\title{
Design, Synthesis and Applications of Retinal-Based Molecular Machines
}

\author{
Diego Sampedro, Marina Blanco-Lomas, \\ Laura Rivado-Casas and Pedro J. Campos \\ Departamento de Química, Unidad Asociada al C.S.I.C., Universidad de La Rioja \\ Spain
}

\section{Introduction}

Progress of mankind has always been related to the development and construction of new machines. In the last decades, science and technology have been involved in a race to increase the capacity of novel machines as well as in a progressive miniaturization of their parts. Further efforts to design and construct machines at the nanometer scale will lead to new and exciting applications in medicine, energy and materials. However, until now every attempt to build artificial systems at the molecular level with complex functions pales beside the Nature's molecular machines at work. Myosin and kinesin enzymes responsible of muscle contraction, ATP synthase and cellular transport are all examples of Nature's ability to provide living systems with complex machinery whose structures and detailed mechanisms we are just starting to unveil. Thus, by learning from Nature, we will be able to make use of the excellent properties refined by slow evolution.

When we mimic Nature, we try to duplicate some of the features found in biological systems using synthetic analogues. Taking natural molecular machines as a starting point, we will try to design, synthesize and explore biomimetic artificial machines. Located at the interface between biology, physics and chemistry, the task of mimicking Nature's results will need combined efforts from different disciplines and the use of every possible tool from theoretical calculations to advanced synthetic chemistry and structural characterization.

In this chapter we will briefly review some of the better-known natural molecular machines as an inspiration for the design of biomimetic artificial machines. Specifically, the structure and function of the retinal molecular machine will be discussed. Taking the Nature's work as a starting point, we will specify some of the requirements to build efficient molecular machines, such as controlling the motion at the molecular level and the energy supply. We will use these concepts to design a set of retinal-based biomimetic chemical switches. Comparison between the synthetic and biological structures allows to gather a better understanding of both systems together with some suggestions for further improvements. Some practical applications will also be presented together with an outlook for the near future.

\section{Why mimic Nature's work?}

Science ability to design, build and manipulate devices of increasing complexity has allowed mankind to reach and occupy every corner of Earth. We are now able to fly through air and 
to cleave through the waves. We have developed new materials with enhanced properties. We have built machines capable of performing complex functions. However, we should bear in mind that Nature had solved most of these problems time ago (Ball, 2001). Even more, Nature solutions are usually more complex, elegant and efficient that the human equivalents. For example, some natural materials are designed to be hard and strong enough to protect living organisms, such as those forming shells and bones (Smith, 1999). Beyond their excellent mechanical properties (Wainwright, 1982), these materials are usually the most economic choice from a biological point of view, thus allowing the living organism to save energy and components for other important biological functions. Mankind has taken advantage of natural materials, but always has tried to emulate or improve Nature's design. For instance, silk is one of the strongest natural fibers. It is made up of the aminoacid series Gly-Ser-Gly-Ala forming beta-sheets with hydrogen bonds between chains. The high proportion of glycine allows the fibers to be strong and resistant to stretching. Thousands of years ago, Chinese recognized the remarkable properties of silk and tried to emulate them looking for an artificial silk (Kaplan \& Adams, 1994). However, it wasn't until 1890's when the first artificial silk (viscose) was produced from cellulose. With the emerge of bionics in the $20^{\text {th }}$ century, research on systems based upon or similar to those of living organisms allowed new types of bioengineered devices. A rapid growth in interest followed on learning how living systems achieve high degrees of organization, synthesize materials with exceptional properties and develop complex devices to interact with the environment.

Scientists have drawn their bioinspiration in two main ways. On one hand, a biological system could be used in a synthetic system as is. Using this approach, the system's functionality is transferred to an artificial construction in order to use its properties in a new way, even completely diverse from its original one (Willner 2002). For instance, DNA has been employed in recent years for new and exotic uses, very different from its biological role such as using selective bonds between complementary DNA sequences to link particles to surfaces. On the other hand, Nature's work can be emulated trying not only to use or understand how biological systems work, but also to use them in artificial devices with new and improved properties (Sarikaya \& Aksay, 1993; Mann, 1996). Taking biological systems as a starting point, scientists try to identify the key factors behind their structure and function to build new systems with different, improved or more controllable properties.

Nature has also developed great examples of efficient machinery. Over millions of years of refinement, living organisms show a number of biomechanical machines much more capable than our synthetic prototypes. Responsible of innumerable biological processes, these biomolecular motors and machines are nanoscale versions of macroscopic machines that we use every day. From these biomolecular machines, we could learn how to efficiently design our own versions of nanoscopic devices. Our goal could be to mimic Nature at first and, why not, try to improve the properties of these systems or at least to adapt them to our specific needs. In the next section we will briefly present some natural machines. Learning how these machines work, we will be able to design and build biomimetic artificial machines exploiting the slow evolutionary Nature's work.

\section{Natural molecular motors}

The way macroscopic machines and motors are regarded can be extended to a molecular level (Balzani et al., 2008). In the last 50 years, Nanotechnology has advanced in the study of machines at the microscopic grade, which are constructed by a "bottom-up" approach. 
However, different examples of nanoscale machinery can be found in Nature. Specifically, cells house hundreds of different molecular machines and motors, each of them specialized for a particular function. These nanomachines are primarily composed of proteins, nucleic acids and other organic molecules. In order to work, energy is needed, so these natural molecular machines and motors convert chemical energy stored in chemical bonds or gradients across membranes into mechanical energy. They are involved in a multitude of essential biological processes, such as transport of cations $\left(\right.$ i.e. $\left.\mathrm{H}^{+}, \mathrm{Ca}^{+}, \mathrm{K}^{+}\right)$, synthesis of ATP, and muscle contraction. In the following paragraphs, different examples of natural molecular machines and motors are described.

\subsection{Natural metal ion channels}

Cells require the passage of cations such as $\mathrm{Na}^{+}, \mathrm{Ca}^{+}$and $\mathrm{K}^{+}$, across their membranes, so they can be distributed to their components. However, this process is prevented by the existence of membranes that protect the contents of cells. Therefore, cation transfer has to take place either by carriers or through ion channels. Carriers are hosts molecules that are embedded in the membrane and help cations to go through the membrane by means of complexation. The rate of this transport mechanism is relatively slow, because it is limited by diffusion. On the other hand, ion channels are membrane proteins that form aqueous ion-conduction pathways through the center of the protein and expand the cell membrane so the ions can move across. These protein channels are found to transport ions faster than a carrier. As an example of a natural ion channel, the mechanism of a light driven proton pump, bacteriorhodopsin, a membrane protein of the halophilic microorganism Halobacterium salinarum is described below (Subramaniam \& Henderson, 2000; Kühlbrandt, 2000).

Bacteriorhodopsin, consists of seven membrane-spanning helical structures linked by short loops on either side of the cell membrane. It also contains one molecule of a linear pigment called retinal that is covalently attached to the protein via a protonated Schiff base. The retinal chromophore, which will be further studied in section 3.5, suffers an isomerization from all-trans to 13-cis upon illumination. This structural change is used by the Schiff base to push a single proton through the seven-helix bundle, from the inside of the cell to the extracellular medium, being subsequently reprotonated from the cytoplasm. Therefore, in this particular movement, the retinal chromophore acts as a valve inside of the cell membrane of this organism.

\subsection{ATP synthase}

As it was stated before, energy is required in order for the molecular machines and motors to work. They are usually fueled by the energy stored in cells. Two of the most common energy repositories of cells are in the phosphate bonds of nucleotides, generally ATP (adenosine triphosphate), and in transmembrane electrochemical gradients. Synthesis of ATP is carried out by the enzyme ATP synthase, which is a natural rotary motor that uses both kinds of the energy sources mentioned above (Metha et al., 1999). This protein is a multidomain complex consisting of two units attached to a common shaft: a hydrophobic proton channel $\left(\mathrm{F}_{0}\right)$ embedded in the mitochondrial membrane and a hydrophilic catalytic unit $\left(\mathrm{F}_{1}\right)$ protruding into the mitochondria. The complex can be thought of as two rotary motor units coupled together. The $\mathrm{F}_{1}$ motor uses free energy of ATP hydrolysis to rotate in one direction whereas the $\mathrm{F}_{0}$ motor uses the energy stored in a transmembrane electrochemical gradient to turn in the opposite direction. The $\mathrm{F}_{1} \mathrm{~F}_{0}-\mathrm{ATP}$ synthase is reversible; 
whereas the full enzyme complex can synthesize or hydrolyze ATP, $F_{1}$ in isolation only hydrolyzes it. This depends on the driven force of the movement. When $\mathrm{F}_{0}$ takes over, which is the normal situation, it drives the $F_{1}$ motor in reverse producing the synthesis of ATP from $\mathrm{ADP}$ and inorganic phosphate. However, when $\mathrm{F}_{1}$ motor controls the rotation, it drives the $\mathrm{F}_{0}$ motor in reverse, becoming an ion pump that moves ions across the membrane against the electrochemical gradient. Rotation of $\mathrm{F}_{1}$ was demonstrated by directly observing the motion of a fluorescent actin filament specifically bound to the rotor element (Noji et al., 1997). Also, discrete $120^{\circ}$ rotations were observed under low ATP concentrations and with actin filaments of variable length (Yasuda et al., 1998). Moreover, they estimated the work required to rotate the actin filament against viscous load to be as much as $80 \mathrm{pN} \mathrm{nm}$, which is approximately the free energy liberated by a single ATP hydrolysis under physiological conditions. Therefore, they concluded that the $\mathrm{F}_{1}$-ATPase could couple nearly $100 \%$ of its ATP-derived energy into mechanical work, so it was considered a really efficient motor.

\subsection{Kinesin and myosin}

Linear-like movements are essential in Nature, because they are related to intracellular trafficking, cell division and muscle contraction (Goodsell, 1996; Howard, 2001). Therefore, one of the main classes of biomolecular motors is linear motors. These are organic molecules or molecular assemblies which move in a linear fashion along a track of some kind. The first type of linear motor is a processive motor, which is constantly in contact with the track it moves along. Processive motors are exemplified by the kinesin protein super-family that moves along microtubules (Schliwa \& Woehlke, 2001). RNA polymerase, which synthesizes new RNA from a single strand RNA template (Gelles \& Landick, 1998), and DNA helicase, which translates along and unwinds DNA in preparation for new DNA synthesis (Lohman et al., 1998), are also linear processive motors.

In addition to processive motors, there are also non-processive motors, which detach from the track and subsequently re-attach, and therefore can be seen as hopping along the track instead of walking. Non-processive linear motors include myosin (Yildiz et al., 2003), which binds to actin filaments and generates the contractile force in muscle tissue (Irving et al., 1992), as well as the dynein protein family that transports cargo along microtubules in the opposite direction to kinesin (Taylor \& Holwill, 1999).

Conventional kinesin is a protein assembly whose total size is approximately $80 \mathrm{~nm}$. It is composed of two larger protein chains, which are involved in microtubule binding, mobility, ATP hydrolysis and protein dimerization, as well as two smaller protein chains, which regulate heavy chain activity and binding to cargo. Kinesin transports cargo along microtubules, self assembled from monomeric proteins, using a walking-like motion with 8 nm steps (Svoboda et al., 1993). Each of these steps is coupled to the hydrolysis of an ATP molecule, which provides the chemical energy for motion (Coy et al., 1999). It moves with a high speed of about $1.8 \mu \mathrm{m} \mathrm{s}^{-1}$, and can move against loads of $6 \mathrm{pN}$. Kinesin is one of the most widely studied motor proteins, and it can be easily modified by genetic engineering and incorporated into a variety of synthetic systems. Microtubules may be bound to a substrate while retaining their structure and function (Turner et al., 1995). Then, molecules functionalized with kinesin can be shuttled along the tracks by the addition of ATP (Diez et al., 2003). It is possible to align microtubules by fluid flow, allowing the kinesin-powered cargo to move in a directional fashion.

The term myosin refers to at least 14 classes of proteins, each containing actin-base motors. Myosin is composed of two large heads, containing a catalytic unit for ATP hydrolysis, 
connected to a long tail (Metha et al., 1999). Myosin II (skeletal muscle myosin) provides the power for all our voluntary motion (running, walking, etc.) and involuntary muscles (i.e. beating heart). In muscle cells, many myosin II molecules combine by aligning their tails, each staggered relative to the next. These muscle cells are also filled with filaments of actin (helical polymers), which are used as a ladder on which myosin climbs. The head groups of myosin extend from the surface of the resulting filament like bristles in a bottle brush. The bristling head groups act independently and provide the power to contract muscles. They reach from the myosin filament to a neighboring actin filament and become attached to it. Breakage of an ATP molecule then forces the myosin head into a radically different shape. It bends near the center and drags the myosin filament along the actin filament. This results in the power stroke of muscle contraction. In a rapidly contracting muscle, each myosin head may stroke five times a second, each stroke moving the filament approximately $10 \mathrm{~nm}$. Besides muscle contraction, myosin II is also involved in several forms of cell movement, including cell shape changes, cytokinesis, capping of cell surface receptors, and retraction of pseudopods (Spudich, 1989). Myosin II shares many structural features with kinesin. Both use ATP to move along their respective tracks, but as it was said in previous paragraphs, myosin II is a non-processive motor, which means that a single molecule cannot move along its track for large distances so organized ensembles of molecules can move their track at higher speeds. Myosin is thought to undergo a conformational change when it binds to actin, resulting in a "working stroke."

The myosin-actin system can also be used to produce the same effect as the one described for kinesin (Harada et al., 1997). As myosin is larger than kinesin, and actin filaments are more flexible than microtubules, more freedom is allowed in the design of synthetic systems.

\subsection{Other molecular machines}

In addition to linear and rotary motors, Nature has devised many other types of nanomachines, such as:

- Springs: The spasmoneme supra-molecular spring is an example of a biological version of a spring (Mahadevan \& Matsudaira, 2000). Thanks to the binding and removal of calcium these filaments cause a reversible contraction and extension that is used by the organism for his protection.

- Hinges: Some proteins, such as the maltose-binding protein, have been found to undergo hinge-like conformational changes when binding a ligand. As a result, a very sensitive maltose sensor can be constructed in vitro (Benson et al., 2001).

- Spindles: The mechanism that some viruses use for packaging their DNA into the viral capsid is analogous to a spindle used for spinning yarn.

- Electrostrictive materials: Presin is a motor protein that resides in the inner ear, whose shape responds to changes in electrical potential across membranes (Liberman et al., 2002). Prestin's electroselective mechanism is responsible for sound amplification and results in a 1000-fold enhacement of sound detection.

\subsection{Retinal chromophore}

One of the most remarkable examples in Nature of a molecular motor is the retinal chromophore of rhodopsin, which suffers a cis-trans photoisomerization during the process of vision (Kandori et al., 2001). Rhodopsin, is a photoreceptor protein (a visual pigment), which is located in the rod visual cells responsible for twilight vision. Rhodopsin has 11-cis retinal as its chromophore (Figure 1), which is embedded inside a single peptide transmembrane protein called opsin. The role of rhodopsin in the signal transduction cascade of vision is to activate 
transducin, a heterotrimeric $G$ protein, upon absorption of light (Hofmann \& Helmreich, 1996). Rhodopsin (opsin), a member of G-protein coupled receptor family, is composed of 7transmembrane helices. The 11-cis retinal forms the Schiff base linkage with a lysine residue of the 7th helix (Lys296 in the case of bovine rhodopsin), and the Schiff base is protonated, which is stabilized by a negatively charged carboxylate (Glu113 in the case of bovine rhodopsin). The bionone ring of the retinal is coupled with hydrophobic region of opsin through hydrophobic interactions (Matsumoto \& Yoshizawa, 1975). Thus, the retinal chromophore is fixed by three kinds of chemical bonds in the retinal binding pocket of rhodopsin.

In the absence of visible light, retinal presents a cis conformation between its carbons 11 and 12. However, when 11-cis-retinal absorbs visible light $(\lambda$ between 400-700 nm), photoisomerization of this double bond takes place to give a trans conformation. Picosecond time-resolved spectroscopy of 11-cis locked rhodopsin analogs reveals that the cis-trans isomerization of the retinal chromophore is the primary reaction in the process of vision, and it is followed by a conformational change in the rhodopsin protein, which generates an electric impulse that reaches the brain, so objects and images can be perceived.

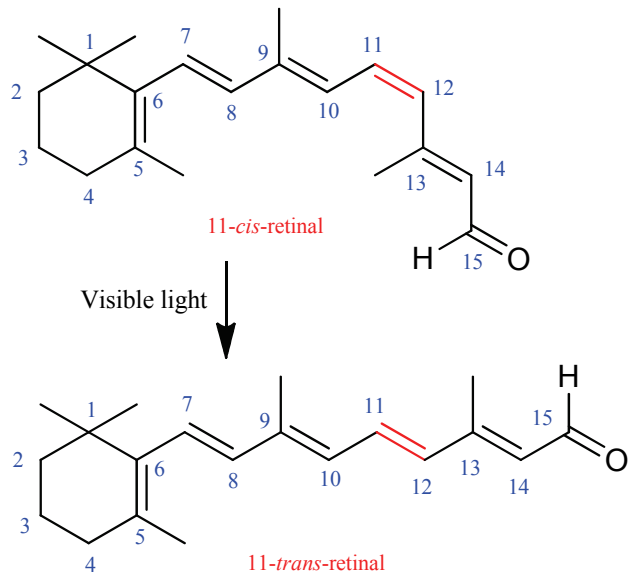

Fig. 1. Chemical structure of the retinal chromophore in rhodopsin

It is known that the cis-trans isomerization is highly efficient in rhodopsin (quantum yield, $\Phi_{\text {isom }}=0.67$, Dartnall, 1967), which is essential to make twilight vision highly sensitive. In fact, a human rod cell can respond to a single photon absorption. Such an efficient photoisomerization of the retinal chromophore is characteristic in the protein environment of rhodopsin, being in contrast to the rhodopsin chromophore in solution. This indicates that the protein environment facilitates the isomerization.

In order to mimic Nature's molecular machines, the molecular structure of the retinal chromophore may be used as a pattern for the design of new prototypes of molecular motors, whose movement is based on cis-trans photoisomerizations. Therefore, molecular motors that work efficiently may be obtained.

\section{Artificial molecular machines}

As stated before, two different approaches can be adopted in order to design practical biomimetic molecular machines. The first approximation is to introduce natural molecular 
machines, such as those shown in section 3, into artificial devices. This way, new hybrid machinery could be built combining the efficiency of natural machines with new applications and uses of these hybrid devices. In fact, several examples of this approach have already been developed (Steinberg-Yfrach et al., 1998; Soong et al., 2000; Hess et al., 2004). The other possibility is to use the natural systems as an inspiration and starting point, trying to adjust their properties to specific needs or even trying to improve their performance. In order to design efficient artificial molecular machines, some key factors should be considered. For instance, some basic questions such as size, medium of operation, type of motion and time scale, to cite a few should be carefully considered in the design. Especially important is the energy supply to make the machine work. In this section we will summarize some of these factors.

\subsection{Basic concepts}

Molecular machines (both natural or artificial) are devices designed to accomplish a specific function. This function is achieved by converting energy into mechanical work. Molecular devices operate via electronic or nuclear rearrangement that have to be controlled beyond the Brownian motion (Astumian, 2005).

As explained in previous paragraphs, different types of motion may be performed by the machine components (oscillatory, linear, rotatory,...). Therefore, the machine should be carefully designed in order to maximize the desired movement while minimizing other competitive motions that would diminish efficiency of the machine. For instance, rotary movement might be achieved using rotations around covalent bonds.

However, not only is important to control the movement of the components of the machine, but also to monitor this movement. In order to achieve this, the electronic or nuclear rearrangements should cause a change in a physical or chemical property that could be measured. In particular, we will see later how the use of light is especially convenient as it can be used both to operate the machine and to monitor the state of the system.

Moreover, a machine capable of cyclic process will be much easier to control and operate. In the case of devices unable to repeat the operation, an external stimulus different from the energy input, should be used to reset the system. This will clearly contribute to slow down the machine operation and increase the complexity of the system. On the other hand, a machine that can operate in a cyclic way could become autonomous, which means that it would keep operating in a constant environment as long as the energy source is still available. Most of the natural machines are autonomous while the majority of artificial devices need a reset. This is one of the main advantages of natural devices that artificial analogues should try to mimic.

Finally, the time scale of the process is also relevant. The time of operation of a molecular machine depends on the type of rearrangement, the components involved and the medium surrounding the machine. The time scale can range from picoseconds to seconds. Thus, other properties such as the type of motion and the property to be monitored should also be in a similar time scale.

\subsection{Energy supply}

A key factor in the design of efficient molecular machines is the energy supply. As said before, molecular machines act through rearrangements caused by suitable stimuli that eventually convert energy into mechanical work. The nature of these stimuli determines not only the chemical nature of the machine, but also the type of motion and the control of the movement (Balzani, 2008). As we have seen in the previous section, most of natural 
machines are activated by chemical stimuli. Proton concentration, ion gradients or interaction with molecules can affect (either activating or deactivating) the machine's operation. However, for a machine activated by chemical energy, it has to be considered also the need for an effective removal of waste products formed during the machine's operation. This fact implies serious limitations in the design and function of artificial molecular machines based on chemical stimuli (Khramov et al., 2008).

Perhaps the simplest stimulus to activate a machine is temperature. For example, the activity of an enzyme can be seriously affected by a temperature increase causing small conformational changes (Min et al., 2005). However, using heat as the energy supply has some serious drawbacks as it is quite difficult to control (both in terms of time and location) and heat dissipates quite rapidly.

Even though it is difficult to employ mechanical forces as an adequate stimulus in artificial systems, this kind of energy supply can be observed in natural devices. For instance, the sense of hearing and touch rely on the effect of mechanical forces.

The ability of electrochemical inputs to produce endergonic and reversible reaction has also been exploited in order to design devices activated by these inputs (Kaifer \& Gómez-Kaifer, 1999). Therefore, heterogeneous electron transfer processes can be used to operate molecular machines with some advantages, such as the absence of waste products to remove and the allowance of electrodes of a very efficient interface between the molecular-level device and the macroscopic world.

Finally, light is probably the most advantageous stimulus as it lacks most of the drawbacks shown by other types of energy inputs. There are no waste products, it is easily controlled by modern optical apparatus, it shows high temporal and spatial resolution with the use of lasers, and precise selection of wavelength allows the selective operation of the device in complex media. Photochemical inputs can be used at the same time to operate and control the machine motion, which facilitates both the design and function of the device. To be more specific, probably the simplest and most used type of reaction to activate a light-driven molecular device is an isomerization reaction.

\section{Retinal-based molecular switches}

We have seen in previous sections how the combination of advanced synthesis, supramolecular chemistry, surface science and molecular biology can provide exciting opportunities toward the development of smart molecular materials and machines (Feringa, 2007). In this section we will review some of the work done on the artificial molecular switches based in the retinal chromophore, one of the best natural examples of efficient molecular machinery.

\subsection{Basic features and design}

Molecular switches and motors are essential components of artificial molecular machines. In fact, switchable molecular systems are molecules which respond to external stimuli and constitute several examples of how simple concepts can be built upon to yield properties with a very wide range of applications. In 1999, a biarylidene molecule was synthesized that uses chemical energy to activate and bias a thermally induced isomerization reaction, and thereby achieve unidirectional intramolecular rotary motion. This one was the first example of a molecular motor able to do photo-induced isomerizations repetitive and unidirectional. (Komura et al., 1999). 
Many advances have occurred from that moment in order to improve the performance of these nanostructures (Vicario et al., 2006). Special emphasis is given to the control of a range of functions and properties, including luminescence, self-assembly, motion, color, conductance, transport, and chirality. Currently, the design and preparation of molecular switches, i.e. molecules that can interconvert among two or more states, based on photochemical cis/trans isomerization constitute an attractive research target to obtain novel materials for nanotechnology (Amendola, 2001; Drexler, 1992; Balzani et al., 2008). Indeed, switches based on the photoisomerization of the azobenzene chromophore have been already used to control ion complexation (Shinkai et al., 1983), electronic properties (Jousselme et al., 2003) and catalysis (Cacciapaglia et al., 2003) or to trigger folding/unfolding of oligopeptide chains (Bredenbeck et al., 2003). Most remarkably, a sophisticated application of the above principle led to the preparation of light-driven molecular rotors (Feringa, 2001) where chirality turned out to be an essential feature to impose unidirectional rotation. In these systems helical bis-arylidene scaffolds featuring a single exociclyc double bond have been employed to achieve photo-induced unidirectional rotary motion. Thanks to the structural changes in these compounds, the rotational velocity is now comparable with those natural ones (i.e. ATP-synthase, see section 3). Among the most natural amazing examples is the cis / trans isomerization of retinal chromophore (rhodopsin protein) in the process of vision (Figure 2) (Gennadiy et al., 2005).
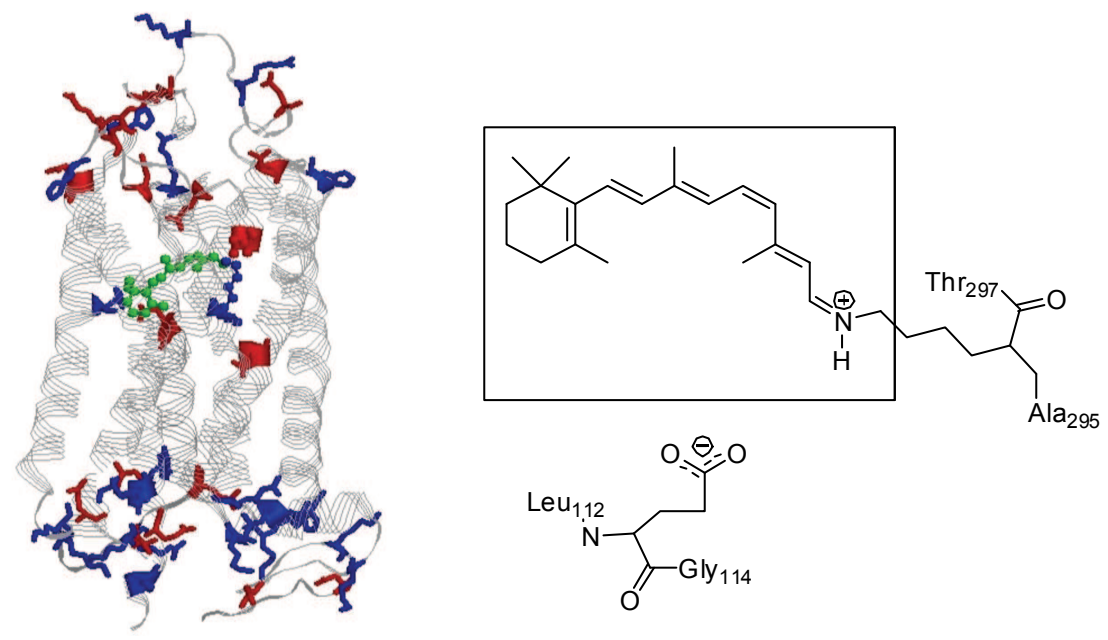

Fig. 2. Rhodopsin protein and retinal chromophore.

As said before, rhodopsin $(\mathrm{Rh})$ is a red-coloured protein due to the 11-cis-retinal. The chromophore is bound to the hydrophobic core of the molecule, causing its absorption maximum at approximately $380 \mathrm{~nm}$. The chromophore is covalently linked to Lys 296 in bovine rhodopsin through a protonated Schiff base (PSB11, Teller et al., 2001). The process of vision takes places due to the cis-trans isomerization which is activated by photons of visible light (400-700 nm). This molecular movement triggers an electric impulse to the brain in femtoseconds. Due to the high efficiency of retinal in vivo isomerization, it has been comprehensively studied and used as a model to the design of many light driven molecular switches. While it has been established that the efficiency of the PSB11 isomerization 
reaction is enhanced by the complex protein environment, an interesting modification is to design a non-natural protonated Schiff base (PSB) capable of replicating in solution the properties of the protein-embedded chromophore. Thus, PSBs of polyenals constitute a class of light-driven switches selected by biological evolution that provide a useful model for the development of artificial light driven molecular switches or motors.

In spite of the increasing interest in these systems, few works of switches based in this mechanism have appeared in literature. In 2004, a new family of retinal-based molecular switches was designed and synthesized (Sampedro et al., 2004). These molecular systems based in the retinal chromophore included an $N$-alkylated pirroline (NAP) moiety and presented some of the features of an efficient light-driven molecular switch (Figure 3).
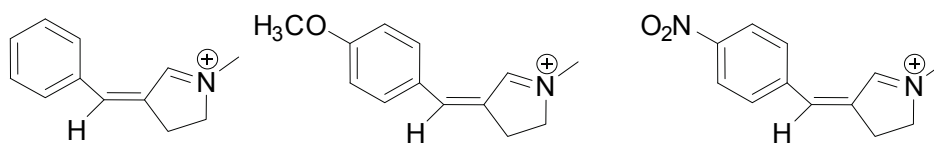

Fig. 3. N-alkylated pirroline (NAPs) molecular switches.

NAPs and other derivates are "chimerical" switches that incorporate into the Feringa's biarylidene skeleton a protonated or alkylated Schiff base function that could potentially replicate the dynamics of the PSB11 isomerization in Rh. One of the characteristic features of these compounds is the quaternization of nitrogen atom, searching for a similarity to the retinal chromophore in vivo. However, one of the principal problems of NAP switches is their low quantum yield $\left(6 \times 10^{-3}\right)$. This result is due to the fact that some energy is not used in the photoisomerization process, but in the isomerization of other bonds different from the central double bond. In general an effective light-driven molecular switch should optimize their performance in order to use all the light energy in a mechanical, controlled way.

Thus, among other important features, an efficient molecular switch must have in its structure a double bond capable of selective photoisomerization in the presence of other bonds. Using the retinal chromophore as a starting point and inspiration, the challenge is to design and synthesize systems that can act as light-driven molecular switches.

\subsection{Synthesis}

A set of molecular switches with the NAPs substructure was synthesized using the following route (Figure 4):

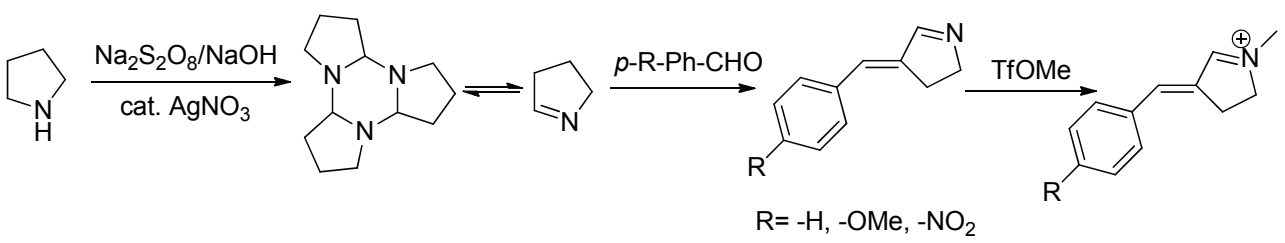

Fig. 4.

The main drawback of these compounds is the presence of different bonds able to isomerize. Due to this, the quantum yield of isomerization around the central double bond is quite low. In order to solvent this problem, a new type of compound with an $N$-alkylated fluorenylidenepyrroline (NAFP) substructure was designed. Isomerization in these compounds is selective as 
only the $\mathrm{C}=\mathrm{C}$ central bond can rotate. Besides, the extended conjugated $\pi$ system allows for absorption bands in the visible (Figure 5, Rivado-Casas et al., 2009).

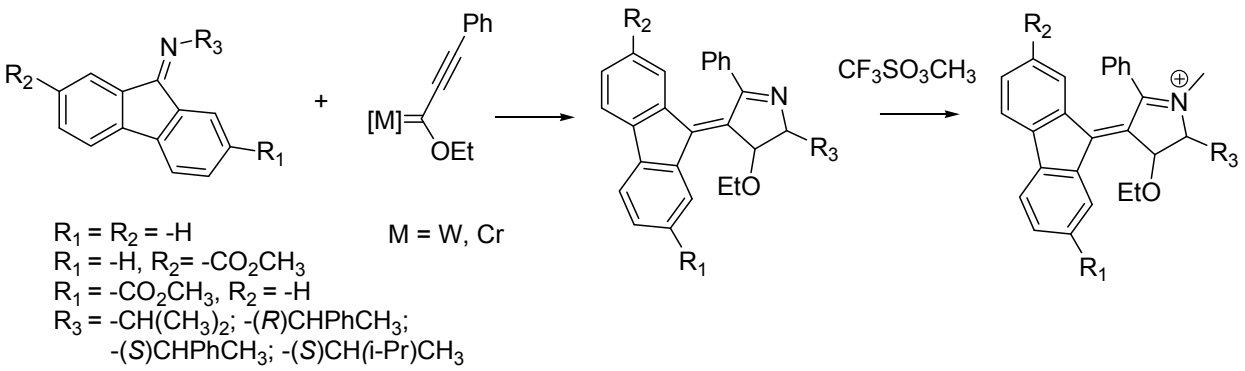

Fig. 5. NAFP light-driven molecular switches.

This new family satisfies some of the features of an ideal molecular switch:

i. selective isomerization (five-membered rings block competitive isomerizations);

ii. high quantum yield;

iii. linking points present in the molecule.

Using NAFPs it is possible to achieve biomimetic light-driven switches where the absorption maximum of an unsubstituted system has been red-shifted by $>50 \mathrm{~nm}$ with respect to that of the NAPs and rotation is now easily achieved by using visible light.

The new structures are one type of biomimetic light-driven Z/E molecular switches that constitute an attractive alternative (e.g. with higher polarity and reduced molecular size) to the previously used azobenzene switch. It should be noted that the cis/trans nomenclature used in the case of the retinal chromophore becomes ambiguous when referring to NAFPs. In this case the use of the $Z / E$ terminology is necessary, but both refer to the same isomerization reaction. Another advantage was the synthesis of chiral molecular switches potentially acting as molecular motors. Moreover, the chiral compounds constitute one of the first examples of a potential light-driven biomimetic single molecule motor whose $Z \rightarrow E$ and $E \rightarrow Z$ rotation direction are ultimately determined by the absolute configuration of the stereogenic centre.

A different group of switches with an $\mathrm{N}$-alkylated indanylidene-pyrroline (NAIP) moiety were also synthesized (Lumento et al., 2007). These compounds display in solution properties similar to the Rh-embedded PSB11 (Figure 6).

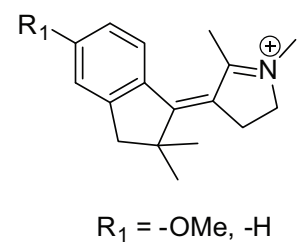

Fig. 6. NAIP molecular switches.

All these compounds (NAPs, NAFPs y NAIPs) show features that in some cases clearly improve those shown by the most popular azobenzenes. Activation may be achieved using low-energy vision light (NAFPs), the length of the indane-pyrroline framework is shorter and may be more easily integrated in a biological compound, such as in a peptide backbone, and they all have good photochemical and mechanical properties. Therefore, they are appropriate candidates to act as molecular light driven switches. 


\subsection{Properties}

Retinal-based switches incorporate into the biarylidene skeleton a protonated or alkylated Schiff base function that could potentially replicate the dynamics of the PSB11 isomerization in $\mathrm{Rh}$. This protein features a $S_{1}$ lifetime of $150 \mathrm{fs}$, a $S_{0}$ transient (photorhodopsin) appearance time of $180 \mathrm{fs}$, and a primary photoproduct (bathorhodopsin) appearance time of 6 ps (Wang et al., 1994). This ultrafast reaction time scale and vibrational coherence suggests that the reaction occurs without intermediates along the reaction path. These intermediates could divide the proton energy up and so it would decrease the efficiency of the switch. In an efficient switch, the reaction path from the excited state happens without any intermediate, and the excited reactive quickly fall over to the product state.

NAIP derivates have been shown, through a highly interdisciplinary research effort (Sinicropi et al., 2008) to be photochromic compounds completing its $Z \rightarrow E$ and $E \rightarrow Z$ photocycle in picoseconds, a few orders of magnitude faster than the fastest known biarylidene $(\approx 6$ miliseconds for a half-cycle, Vicario et al., 2006). This is in agreement with a reaction path where the formation of the photoproduct takes place without any intermediate, as in the case of the retinal chromophore in vivo. Thus, these biomimetic switches constitute very efficient compounds as they show limited energy redistribution and fast isomerization. Also, these compounds show high thermal interconversion barriers $(>40 \mathrm{kcal} / \mathrm{mol})$. These barriers indicate that the $E$ - and $Z$ - forms will no thermally interconvert at room temperature.

The high efficiency of this photoreaction is a clear improvement over switches based on azobenzene. In rhodopsin, the photoisomerization of the native 11-cis-retinal chromophore to its all-trans form occurs with a 0.67 quantum yield (Dartnall, 1967). This value is larger than the 0.25 value measured for the same chromophore in ethanol solution (Becker, 1988) and also larger than the approximately 0.39 value measured for the photoisomerization of trans- and cis- azobenzene, respectively (Bortolus \& Monti, 1979). However, the quantum yields measured for NAFPs (0.5) are much closer to retinal chromophore in vivo. This behaviour is also in agreement with a reaction path without intermediates.

One of the most remarkable properties of NAFPs is that these switches display absorption maxima in the near visible region (Rivado-Casas et al., 2009). Specifically, they have an absorption maximum of $c a .400 \mathrm{~nm}$ that is red-shifted compared to the NAPs derivates (Lumento et al., 2007). This displacement is due to the replacement of the pyrroline unit of NAPs with moieties displaying an expanded п system. This constitutes a clear improvement over previous results and makes NAFP retinal-based molecular switches interesting candidates for technological applications.

NAFP switches show also high stability, both thermal and photochemical. In fact, the degree of photodegradation was assessed irradiating the switches. It was found that the $1 \mathrm{H}-\mathrm{NMR}$ spectrum remained unaltered after long times ( $>24$ hours), showing that the photochemical decomposition of these molecules is minimal.

To summarize:

i. these switches are suitable for applications in highly polar environments while azobenzene derivates are neutral with limited dipole-moment values;

ii. the length of the indane (or fluoren-pyrroline) framework is shorter that those of azobenzenes and may be more easily integrated, for instance, in a peptide backbone.

iii. the photoisomerization of these switches happens in picoseconds ( $10^{3}$ times faster than azobenzene);

iv. their quantum yield is closer to retinal. 


\subsection{Applications}

The use of light to trigger changes in molecular systems has great importance in biochemistry and medicine (Gorostiza \& Isacoff, 2008). Systems based on the reversible photoreactions of diarylethenes have advanced the area of "smart" materials. Their potential use as components of molecular electronics, optical memories and variable-transmission filters has been well documented (Irie, 2000). Their appeal is based on the fact that light can be tuned to reversibly and specifically trigger optical and electronic changes such as colour, emission and refractive index in materials that contain this versatile molecular architecture.

What are the potential applications of molecular switches, motors and machines is a question asked frequently in the context of future technology based on (molecular) nanoscience. A glance at the myriad of molecular machines on biology and the fascinating diversity of processes is perhaps the best testimony to the prospects ahead. The first examples of molecular motors and switches at work have already been reported (Browne \& Feringa, 2006; Kay et al., 2007; Stoddart, 2001; Harada, 2001). The fascinating idea of casting single molecules capable to convert light energy into "mechanical" motion prompted chemists to develop, in different contexts, systems capable of undergoing light-driven structural changes.

In fact, molecular switches based on photochemical $\mathrm{Z} / \mathrm{E}$ isomerization have been employed in different contexts to convert light energy into "mechanical" motion at molecular level (Sauvage, 2001). In basically all applications, the induced motion results in a permanent or transient conformational change of a molecular scaffold bounded to the switch. The spatial asymmetry (i.e. the chirality) of the molecular framework of NAFPs, NAIPs and NAPs determinates the direction (either clockwise or anticlockwise) of the $E \rightarrow Z$ and $Z \rightarrow E$ conformational changes leading, after sequential abortion of two photons, to a complete $360^{\circ}$ rotation.

One of the uses of these systems is their union to more complex structures. In fact, the analysis and modulation of the conformation and function of biomolecules (e.g. ion transport (Lougheed et al.,2004), protein folding (Fierz et al., 2007), cell signalling (Cordes et al., 2006) and cell adhesion (Schütt et al., 2003) with photochromic switches is an area of increasing interest (Duvage \& Demange, 2003). Among the photoisomerizable subunits for the photomodulation of secondary structure elements in peptides and proteins, photosensitive $\omega$-amino acids are highly promising candidates. $\omega$-Amino acids are non-protein amino acids in which amino and acid groups are in opposite sides of a chain (Figure 7).

For instance, hemithioindigo $\omega$-amino acids (HTI) have shown favourable properties to use in biological systems (Cordes at al., 2007). Photoswitchable amino acids can be used to reversibly activate and deactivate a bioactive compound, and are therefore valuable diagnostic instruments to study complex living systems using light. In fact, the structure of biomolecules

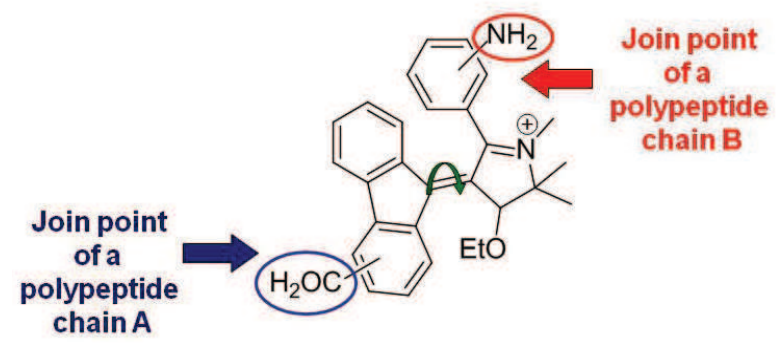

Fig. 7. Use of NAFP switches for protein photomodulation. 
can be modified in a defined way using light, which opens a variety of fascinating new applications in biology and medicine. The potential use of this strategy has been demonstrated and has been already used to control physiological processes in living organisms (Szobata et al., 2007), to activate or modify the expressions of enzymes or to regulate their biological function. Rapid structural changes can also be induced in small biomolecules allowing direct experimental studies of the initial processes in protein folding (Schrader et al., 2007).

\section{Conclusions and outlook}

Through this chapter we have reviewed some natural machines and what we can learn from them in order to design and build our versions of artificial machines. Improving Nature's work is clearly a huge task, but we should not relinquish this intention. A careful exploration of natural machines will lead not only to valuable information on how to design efficient nanoscopic devices, but also will help us to understand how these natural machines work, their advantages and weak points. However, we should bear in mind that biomimetism is more than a slight resemblance to a natural structure. In Nature, the look or structure of biomolecular machine, or any chemical structure in general, is far from being important. Any natural structure responds to the necessity of survival of an organism. Thus, design and synthesis are always subordinated to the accomplishment of this task without any unnecessary expense. Bioinspiration will help us to rapidly improve our nanomachinery, but also the design and construction of new nanoscopic devices and machines will facilitate our understanding of these fascinating examples of natural engineering.

Although the application of retinal-based switches and derivates to the construction of rotary motors will require further research work, computational and spectroscopic data indicate that their photoisomerization dynamics replicates the behaviour of the PSB11 in Rh. A particularly challenging issue is the control of molecular motion as the performance of the motor will be essential in powering future nanoscale machines. The controlled directional movement along a trajectory, the use of molecular motors as transporters for molecular cargo, the construction of motor-powered nanomachines and devices that can perform several mechanical functions and the design of smart switchable surfaces are just a few of the developments that can be envisioned in the near future.

It is evident that this whole field is still in its infancy and offers a wide range of opportunities for molecular design and discovery.

\section{Acknowledgements}

We thank the MICINN, Comunidad Autónoma de La Rioja and Universidad de La Rioja for financial support.

\section{References}

Amendola, V. Structure \& Bonding, Springer, Berlín, 2001.

Astumian, R. D. Proc. Natl. Acad. Sci. U.S.A., 2005, 102, 1843.

Ball, P. Nature 2001, 409, 413.

Balzani, V.; Venturi, M.; Credi, A. Molecular Devices and Machines. Concepts and Perspectives for the Nanoworld, Wiley-VCH, Weinheim, 2008.

Becker, R. S. Photochem. Photobiol. 1988, 48, 369. 
Benson, D.E.; Conrad, D.W.; de Lorimier, R.M.; Trammell, S.A.; Hellinga, H.W. Science, 2001, 293, 1641.

Bortolus, P.; Monti, S. J. Phys. Chem., 1979, 83, 648.

Bredenbeck, J.; Helbing, J.; Sieg, A.; Schrader, T.; Zinth, W.; Renner, C.; Behrendt, R.; Moroder, L.; Wachtveilt, J.; Hamm, P. Proc. Natl. Acad. Sci. U.S.A., 2003, 100, 6452.

Browne, W. R.; Feringa, B. L. Nat. Nanotechnol., 2006, 1, 25.

Cacciapaglia, R.; Stefano, S. D.; Mandolini, L. J. Am. Chem. Soc., 2003, 125, 2224.

Cordes, T.; Heinz, B.; Regner, N.; Hoppmann, C.; Schrader, T. E.; Summerer, W.; RückBraun, K.; Zinth, W. Chem. Phys. Chem., 2007, 8, 1713.

Cordes, T.; Weinrich, D.; Kempa, S.; Riesselmann, K.; Herre, S.; Hoppmann, C.; Rück-Braun, K.; Zinth, W. Chem. Phys. Lett., 2006, 428, 167.

Coy, D.L.; Wagenbach, M.; Howard, J. J. Biol. Chem., 1999, 274, 3667.

Dartnall, H. Visio Res. 1967, 8, 339.

Diez, S.; Reuther, C.; Dinu, C.; Seidel, R.; Mertig, M.; Pompe, W.; Howard, J. Nano Lett., 2003, 3, 1251.

Drexler, K. E. Nanosystems: Molecular Machinery, Manufacturing and Computation, John Wiley \& Sons, New York, 1992.

Duvage, C.; Demange, L. Chem. Rev., 2003, 103, 2475.

Feringa, B. L. Acc. Chem. Res., 2001, 34, 504.

Feringa, B. L. J. Org. Chem., 2007, 72, 6635.

Fierz, B.; Satzger, H.; Root, C.; Gilch, P.; Zinth, W.; Kiefhaber, T. Proc. Natl. Acad. Sci. U.S.A., 2007, 104, 2163.

Gelles, J.; Landick, R. Cell, 1998, 93, 13.

Gennadiy, M.; Ying, C.; Yusuke, T.; Bill, W.; Jiang-Xing, M. Proc. Natl. Acad. Sci., 2005, 102, 12413.

Goodsell, D.S. Our Molecular nature: The Body's Motors, Machines, and Messages, 1996, Copernicus, New York.

Gorostiza, P.; Isacoff, E. Science, 2008, 322, 395.

Harada, Y.; Noguchi, A.; Kishino, A.; Yanagida, T. Biophys. J., 1997, 72, 1997.

Harada, A. Acc. Chem. Res., 2001, 34, 456.

Hess, H.; Bachand, G. D.; Vogel, V. Chem. Eur. J. 2004, 10, 2110

Hofmann, K.P.; Helmreich, E. J. M. Biochim. Biophys. Acta, 1996, 1286, 285.

Howard, J. Mechanics of Motor Proteins and Cytoskeleton, 2001, Sindauer Associates, Sunderland. Irie, M. Chem. Rev., 2000, 100, 1685.

Irving, M.; Lombardi, V.; Piazzesi, G.; Ferenczi, M.A. Nature, 1992, 357, 156.

Jousselme, B.; Blanchard, P.; Gallego-Planas, N.; Delaunay, J.; Allain, M.; Richomme, P.; Levillain, E.; Roncali, J. J. Am. Chem. Soc., 2003, 125, 2888.

Kaifer, A. E.; Gómez-Kaifer, M. Supramolecular Electrochemistry, Wiley-VCH, Weiheim, 1999.

Kandori, H.; Shichida, Y.; Yoshizawa, T. Biochem. (Moscow), 2001, 66, 1197.

Kaplan,D. ; Adams, W. W. Silk Polym.; ACS Symp. Ser. 1994, 544, 2.

Kay, E. R.; Leigh, D. A.; Zerbetto, F. Angew. Chem. Int. Ed., 2007, 46, 72.

Khramov, D.M.; Rosen, E. L.; Lynch, V. M.; Bielawsky, C. W. Angew. Chem. Int. Ed. 2008, 47, 2267.

Komura, N.; Zijistra, R. W. J.; van Delden, R. A.; Harada, N.; Feringa, B. L. Nature, 1999, 401, 15.

Kühlbrandt, W. Nature, 2000, 406, 569.

Liberman, M.C.; Gao, J.; He, D.Z.Z.; Wu, X.; Jia, S.; Zuo, J. Nature, 2002, 419, 300.

Lohman, T.M.; Thorn, K.; Vale, R.D. Cell, 1998, 93, 9.

Lougheed, T.; Borisenko, V.; Henning, T.; Rück-Braun, K.; Wooley, G. A. Org. Biomol. Chem., 2004, 2, 2798.

Lumento, F.; Zanirato, V.; Fusi, S., Busi, E.; Latterini, L.; Elisei, F.; Sinicropi, A.; Andruniów, T.; Ferré, N.; Basosi, R.; Olivucci, M. Angeww. Chem. Int. Ed., 2007, 47, 414. 
Mahadevan, L.; Matsudaira, P. Science, 2000, 288, 95.

Mann, S. Biomimetic Materials Chemistry, VCH, New York, 1996

Matsumoto, H.; Yoshizawa, T. Nature, 1975, 258, 523.

Metha, A.D.; Rief, M.; Spudich, J.A.; Smith, D.A.; Simmons, R.M. Science, 1999, 283, 1689.

Min, W.; English, B. P.; Luo, G.; Cherayil, B. J.; Kou, C.; Xie, X. S. Acc. Chem. Res. 2005, 38, 923.

Noji, H.; Yasuda, R.; Yoshida, M.; Kinosita Jr., K. Nature, 1997, 386, 299.

Piermattei, A.; Karthikeyan, S.; Sijbesma, R. P. Nat. Chem. 2009, 1, 133.

Rivado-Casas, L.; Sampedro, D.; Campos, P. J.; Fusi, S.; Zanirato, V.; Olivucci, M. J. Org. Chem., 2009, 74, 4666.

Rivado-Casas, L.; Campos, P. J.; Sampedro, D. Organometallics, 2010, $29,3117$.

Sampedro, D.; Migani, A.; Pepi, A.; Busi, E.; Basosi, R.; Latterini, L.; Elisei, F.; Fusi, S.;

Ponticelli, F.; Zanirato, V.; Olivucci, M. J. Am. Chem. Soc., 2004, 126, 9349.

Sarikaya, M. and I. A. Aksay, Biomimetics: Design and processing of Materials, American Institute of Physics, College Park, 1993

Sauvage, J.-P. Molecular Machines and Motors, Springer-Verlag, Berlín, London, 2001.

Schliwa, M.; Woehlke, G. Nature, 2001, 411, 424.

Schrader, T. E.; Schreier, W. J.; Cordes, T.; Koller, F. O.; Babitzi, G.; Denschlag, R.; Renner, C.; Dong, S. L.; Löweneck, M.; Moroder, L.; Tavan, P.; Zinth, W. Proc. Natl. Acad. Sci. U.S.A., 2007, 104, 15729.

Schütt, M.; Krupka, S. S.; Alexander, G.; Milbradt, D.; Deindl, S.; Sinner, E.-K.; Oesterhelt, D.; Renner, C.; Moroder, L. Chem. Biol., 2003, 10, 487.

Shinkai, S.; Minami, T.; Kusano, Y.; Manabe, O. J. Am. Chem. Soc., 1983, 105, 1851.

Sinicropi, A.; Martin, E.; Ryasantsev, M.; Helbing, J.; Briand, J.; Sharma, D.; Léonard, J.; Haacke, S.; Canizzo, A.; Chergui, M.; ZAnirato, V.; Fusi, S.; Santoro, F.; Basosi, R.; Ferré, N.; Olivucci, M. Proc. Natl. Acad. Sci. U.S.A., 2008, 105, 17642.

Smith,B.L.; Schaffer, T.E.; Viani, M.; Thompson, J. B.; Frederick, N. A.; Kindt, J.; Belcher, A.; Stucky, G. D.; Morse, D. E.; Hansma, P. K. Nature 1999, 399, 761.

Soong, R. K.; Bachand, G. D; Neves, H. P.; Olkhovets, A. G.; Craighead, H. G.; Montemagno, C. D. Science, 290, 1555.

Spudich, J.A. Cell Regul., 1989, 1,1.

Steinberg, G.; Rigaud, J.L.; Durantini, E. N.; Moore, A. L.; Gust, D.; Moore, T. A.; Nature, 1998, 392, 479.

Stoddart, J. F. Acc. Chem. Res., 2001, 34, 410.

Subramaniam, S.; Henderson, R. Nature, 2000, 406, 653.

Svoboda, K.; Schmidt, C.F.; Schnapp, B.J.; Block, S.M. Nature, 1993, 365, 721.

Szobata, S.; Gorosita, P.; Del Bene, F.; Wyart, C.; Fortin, D. L.; Kolstad, K. D.; Tulythan, O.;

Volgraf, M.; Numano, R.; Aaron, H. L.; Scoot, E. K.; Kramer, R. H.; Flannery, J.; Baier, H.; Trauner, D.; Isacoff, E. Neuron, 2007, 54, 535.

Taylor, H.C.; Holwill, E.J. Nanotechnology, 1999, 10, 237.

Teller, D. C.; Okada, T.; Behnke, C. A.; Palczewski, K.; Stenkamp, R. E. Biochemistry, 2001, 40, 7761.

Turner, D.C.; Chang, C.Y.; Fang, K.; Bradow, S.L.; Murphy, D.B. Biophys. J., 1995, 69, 2782.

Vicario, J.; Walko, M.; Meetsma, A.; Feringa, B. L. J. Am. Chem. Soc., 2006, 128, 5127.

Wainwright, S. A. Mechanical Design in Organisms, Princeton University Press, Princeton, 1982.

Wang, Q.; Schoenlein, R.W.; Petenau, L. A.; Mathies, R. A.; Shnak, C. V. Science, 1994, 266, 422.

Willner, I. Science 2002, 298, 2407.

Yasuda,R.; Noji, H.; Kinosita Jr., K.; Yoshida, M. Cell, 1998, 93, 1117.

Yildiz, A.; Forkey, J.N.; McKinney, S.A.; Ha, T.; Goldman, Y.E.; Selvin, P.R. Science, 2003, 300, 2061. 


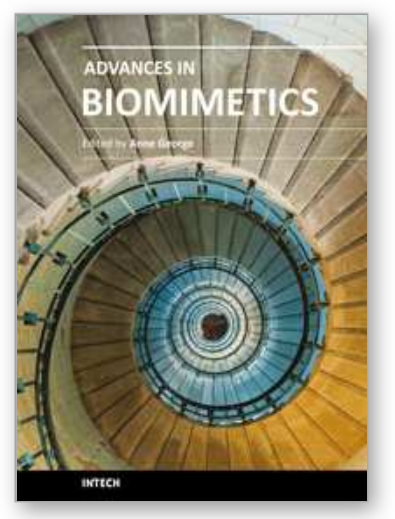

\author{
Advances in Biomimetics \\ Edited by Prof. Marko Cavrak
}

ISBN 978-953-307-191-6

Hard cover, 522 pages

Publisher InTech

Published online 26, April, 2011

Published in print edition April, 2011

The interaction between cells, tissues and biomaterial surfaces are the highlights of the book "Advances in Biomimetics". In this regard the effect of nanostructures and nanotopographies and their effect on the development of a new generation of biomaterials including advanced multifunctional scaffolds for tissue engineering are discussed. The 2 volumes contain articles that cover a wide spectrum of subject matter such as different aspects of the development of scaffolds and coatings with enhanced performance and bioactivity, including investigations of material surface-cell interactions.

\title{
How to reference
}

In order to correctly reference this scholarly work, feel free to copy and paste the following:

Diego Sampedro, Marina Blanco-Lomas, Laura Rivado-Casas and Pedro J. Campos (2011). Design, Synthesis and Applications of Retinal-Based Molecular Machines, Advances in Biomimetics, Prof. Marko Cavrak (Ed.), ISBN: 978-953-307-191-6, InTech, Available from: http://www.intechopen.com/books/advancesin-biomimetics/design-synthesis-and-applications-of-retinal-based-molecular-machines

\section{INTECH}

open science | open minds

\section{InTech Europe}

University Campus STeP Ri

Slavka Krautzeka 83/A

51000 Rijeka, Croatia

Phone: +385 (51) 770447

Fax: +385 (51) 686166

www.intechopen.com

\section{InTech China}

Unit 405, Office Block, Hotel Equatorial Shanghai

No.65, Yan An Road (West), Shanghai, 200040, China

中国上海市延安西路65号上海国际贵都大饭店办公楼405单元

Phone: +86-21-62489820

Fax: +86-21-62489821 
(C) 2011 The Author(s). Licensee IntechOpen. This chapter is distributed under the terms of the Creative Commons Attribution-NonCommercialShareAlike-3.0 License, which permits use, distribution and reproduction for non-commercial purposes, provided the original is properly cited and derivative works building on this content are distributed under the same license. 